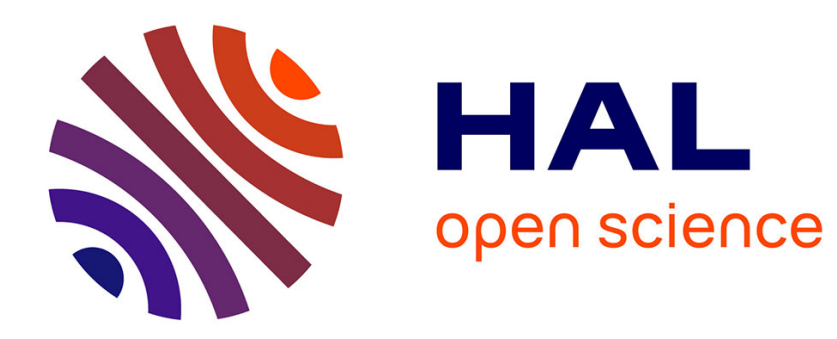

\title{
The total distance for totally positive algebraic integers V Flammang
}

\section{To cite this version:}

V Flammang. The total distance for totally positive algebraic integers. Bulletin of the Australian Mathematical Society, 2014, 90 (3), pp.391-403. 10.1017/S0004972714000537 . hal-01231483

\section{HAL Id: hal-01231483 \\ https://hal.science/hal-01231483}

Submitted on 20 Nov 2015

HAL is a multi-disciplinary open access archive for the deposit and dissemination of scientific research documents, whether they are published or not. The documents may come from teaching and research institutions in France or abroad, or from public or private research centers.
L'archive ouverte pluridisciplinaire HAL, est destinée au dépôt et à la diffusion de documents scientifiques de niveau recherche, publiés ou non, émanant des établissements d'enseignement et de recherche français ou étrangers, des laboratoires publics ou privés. 


\title{
THE TOTAL DISTANCE \\ FOR TOTALLY POSITIVE ALGEBRAIC INTEGERS
}

\author{
V. FLAMMANG
}

\begin{abstract}
Let $P(x)$ be a degree $d$ polynomial with zeros $\alpha_{1}, \ldots, \alpha_{d}$. In 2013, K. Stulov and R. Yang defined the total distance of $P$ as $\operatorname{td}(P)=\sum_{i=1}^{d}|| \alpha_{i}|-1|$. In this paper, using the method of explicit auxiliary functions, we study the spectrum of the absolute total distance for totally positive algebraic integers and find its five smallest points. Moreover, we establish inequalities involving the total distance and two standard measures of totally positive algebraic integers and also the trace of totally positive algebraic integers. We give numerical examples to show that our bounds are quite good. The polynomials involved in the auxiliary functions are found by our recursive algorithm.
\end{abstract}

\section{Introduction}

\subsection{The total distance}

We recall the definitions of two standard measures of polynomials:

let $P=a_{0} x^{d}+a_{1} x^{d-1}+\ldots+a_{d}=a_{0}\left(x-\alpha_{1}\right) \ldots\left(x-\alpha_{d}\right)$ be a polynomial with complex coefficients. Then

- the length of $P$ is $\mathrm{L}(P)=\sum_{i=0}^{d}\left|a_{i}\right|$,

- the Mahler measure of $P$ is $\mathrm{M}(P)=\left|a_{0}\right| \prod_{i=1}^{d} \max \left(1,\left|\alpha_{i}\right|\right)$.

In 2013, K. Stulov and R. Yang [SY] defined a less usual measure, called the total distance of $P$, defined as follows

$$
\operatorname{td}(P)=\sum_{i=1}^{d}|| \alpha_{i}|-1|
$$

If $\alpha$ is an algebraic integer then the measure of $\alpha$ is the measure of its minimal polynomial $P$ in $\mathbb{Z}[x]$. If $\alpha$ is a nonzero algebraic integer and $\mathrm{M}(\alpha)=1$ then a classical theorem of Kronecker $[\mathrm{K}]$ tells us that $\alpha$ is a root of unity. This suggests the question: $\inf _{\alpha \text { not a root of unity }} \mathrm{M}(\alpha)>1$ ? This is known as Lehmer's problem. It is still open. Another formulation of the problem is: does there exist an absolute constant $c>0$ such that: if $\mathrm{M}(\alpha)>1$ then $\mathrm{M}(\alpha)>1+c$ ?

The smallest known value is due to Lehmer himself and is $\mathrm{M}(P)=1.176280 \ldots$ where $P(x)=$ $x^{10}+x^{9}-x^{7}-x^{6}-x^{5}-x^{4}-x^{3}+x+1$.

In their paper, K. Stulov and R. Yang established the following inequalities for a complex polynomial $P$ with $\left|a_{d}\right|=\left|a_{0}\right|=1$ :

$$
\mathrm{m}(P) \leq \operatorname{td}(P) \leq 2(\mathrm{M}(P)-1)
$$

where $\mathrm{m}(P)=\log (\mathrm{M}(P))$. Thus, a third version of Lehmer's question can be formulated as follows: does there exist an absolute constant $c^{\prime}>0$ such that: if $\operatorname{td}(\alpha)>0$, with $\left|a_{d}\right|=\left|a_{0}\right|=1$ then $\operatorname{td}(\alpha)>c^{\prime}$ ? 
In this paper, we are interested in totally positive algebraic integers $\alpha$, i.e., algebraic integers all of whose conjugates are positive real numbers. We define the absolute total distance of $\alpha$ as $\operatorname{Td}(\alpha)=\frac{\operatorname{td}(\alpha)}{\operatorname{deg}(\alpha)}$. As part of a study of the set $\mathcal{T} d$ of such quantities, we prove the following:

Theorem 1. If $\alpha$ is a nonzero totally positive algebraic integer whose minimal polynomial is different from $x-1, x^{2}-3 x+1, x^{3}-5 x^{2}+6 x-1, x^{5}-9 x^{4}+27 x^{3}-32 x^{2}+13 x-1$, $x^{5}-9 x^{4}+28 x^{3}-35 x^{2}+15 x-1$ and $x^{6}-11 x^{5}+45 x^{4}-84 x^{3}+70 x^{2}-21 x+1$ then we have

$$
\operatorname{Td}(\alpha) \geq 1.318853
$$

The polynomials involved in this result can be read from Table 1.

We deduce from this result the first five points of the spectrum of Td which are:

$1.118034 \ldots=\operatorname{Td}\left(x^{2}-3 x+1\right)$,

$1.201291 \ldots=\operatorname{Td}\left(x^{3}-5 x^{2}+6 x-1\right)$,

$1.291483 \ldots=\operatorname{Td}\left(x^{5}-9 x^{4}+28 x^{3}-35 x^{2}+15 x-1\right)$,

$1.312968 \ldots=\operatorname{Td}\left(x^{6}-11 x^{5}+45 x^{4}-84 x^{3}+70 x^{2}-21 x+1\right)$,

$1.317559 \ldots=\operatorname{Td}\left(x^{5}-9 x^{4}+27 x^{3}-32 x^{2}+13 x-1\right)$.

We conjecture that the next smallest point of the spectrum has minimal polynomial $x^{9}-17 x^{8}+$ $120 x^{7}-456 x^{6}+1011 x^{5}-1324 x^{4}+986 x^{3}-376 x^{2}+57 x-1$ and absolute total distance $1.329113 \ldots$

Now, it is natural to compare the total distance with the two standard measures. We establish the following inequalities:

Theorem 2. If $\alpha$ is a nonzero totally positive algebraic integer of degree $d$ whose minimal polynomial is different from $x-1, x-2, x^{2}-3 x+1$ and $x^{2}-5 x+5$ then we have:

$$
0.227804 \mathrm{~m}(\alpha)+1.077638 d \leq \operatorname{td}(\alpha) \leq 86.546972 \mathrm{~m}(\alpha)-43.686533 d \text {. }
$$

Theorem 3. If $\alpha$ is a nonzero totally positive algebraic integer of degree $d$ whose minimal polynomial is different from $x-1, x-2, x-3$ and $x^{2}-3 x+1$ then we have:

$$
0.026162 \operatorname{td}(\alpha)+0.689186 d \leq \log \mathrm{L}(\alpha) \leq 1.084996 \operatorname{td}(\alpha)-0.149578 d .
$$

Let $P=x^{d}+a_{1} x^{d-1}+\ldots+a_{d}=\left(x-\alpha_{1}\right) \ldots\left(x-\alpha_{d}\right)$ be a monic polynomial with complex coefficients. The trace of $P$ is defined by $\operatorname{trace}(P)=\sum_{i=1}^{d} \alpha_{i}=-a_{1}$. Although the trace is not a measure in general, it may be considered as a measure for minimal polynomials of totally positive algebraic integers. So, we can use the trace in our inequalities and we prove:

Theorem 4. If $\alpha$ is a nonzero totally positive algebraic integer of degree $d$ whose minimal polynomial is different from $x-1, x-2, x^{2}-3 x+1, x^{2}-4 x+2, x^{3}-6 x^{2}+8 x-2$ and $x^{3}-6 x^{2}+8 x-1$ then we have:

$$
0.236643 \operatorname{td}(\alpha)+1.307819 d \leq \operatorname{trace}(\alpha) \leq 2.211970 \operatorname{td}(\alpha)-0.635402 d .
$$

In Table 2, Table 3 and Table 4, we give numerical examples where we can see that our bounds are quite good. In particular, Table 2 shows that our bounds are better than those of K. Stulov and R. Yang for the set of polynomials that we consider. For reciprocal polynomials $P$ i.e., $P(x)=x^{\operatorname{deg}(P)} P(1 / x)$, K. Stulov and R. Yang proved the following stronger lower bound: $\operatorname{td}(P) \geq 2 \mathrm{~m}(P)$. We give in Table 5 a set of reciprocal polynomials for which our lower bound is still better than their bound. 


\subsection{Explicit auxiliary function}

The absolute trace of a totally positive algebraic integer $\alpha$ of degree $d \geq 2$ whose conjugates are $\alpha_{1}=\alpha, \ldots, \alpha_{d}$ is $\mathcal{T}$ race $(\alpha)=$ trace $(\alpha) / d=\sum_{i=1}^{d} \alpha_{i} / d$. The Schur-Siegel-Smyth problem is the following: fix $\rho<2$, then show that all but finitely many totally positive algebraic integer $\alpha$ have $\mathcal{T}$ race $(\alpha)>\rho$. The problem was solved by I. Schur in 1918 for $\rho<\sqrt{e}$ [Sc] then by C.L. Siegel in 1943 for $\rho<1.7337$ [Si]. In 1984, C.J. Smyth developed the method of explicit auxiliary functions and solved the problem for $\rho<1.7719$ [Sm]. All subsequent results were obtained thanks to this method. For a complete survey of this problem, see [ABP]. Until 2003, the polynomials involved in the auxiliary functions were found by heuristic methods. In 2003, when studying the linear independence measure of logarithms of rational numbers, $\mathrm{Q}$. Wu $[\mathrm{Wu}]$ developed an algorithm by which the suitable polynomials are found by a systematic search. The author [F2] improved this algorithm in 2006 and now the suitable polynomials are found by induction. The best result for the trace problem to this day is obtained by the author for $\rho<1.792519$ [F4]. The method of explicit auxiliary functions is also used to get the greatest lower bound of the absolute measure of an algebraic integer $\alpha$, all of whose conjugates lie in a sector $S_{\theta}=\{z \in \mathbb{C}$ such that $|\arg z| \leq \theta\}$ with $0<\theta<\pi$. M. Langevin [La] proved that there exists a function $c(\theta)$ on $[0, \pi)$, always $>1$, such that if $\alpha \neq 0$ is not a root of unity, whose conjugates all lie in $S_{\theta}$, then $\Omega(\alpha)=\mathrm{M}(\alpha)^{1 / d} \geq c(\theta)$. Note that this result is not numerical. G. Rhin and C. Smyth [RS] succeeded in finding the exact value of $c(\theta)$ for $\theta$ in nine subintervals of $[0,2 \pi / 3]$ and conjectured that $c(\theta)$ is a "staircase" function of $\theta$, which is constant except for finitely many left discontinuities in any closed subinterval of $[0, \pi)$. The polynomials involved in their auxiliary functions were found by heuristic methods. In 2004, thanks to Wu's algorithm [Wu], G. Rhin and Q. Wu [RW] gave the exact value of $c(\theta)$ for four new subintervals of $[0, \pi)$ and extended four existing subintervals. In 2013, the author and G. Rhin [FR] found for the first time a complete subinterval and a fourteenth subinterval. These improvements are due to our recursive algorithm. The author [F1] computed the greatest lower bound $c(\theta)$ of the absolute trace for $\theta$ belonging to seven subintervals of $\left[0,90^{\circ}\right)$. Q. Wu and L. Wang [WW] used the method of auxiliary functions to improve the irrationality measure of the number $\log 3$.

In Section 2, we explain the method of explicit auxiliary functions. We link it with a generalization of the classical integer transfinite diameter. Then we detail how our recursive algorithm enables us to get the constant of Theorem 1 for the total distance. In Section 3, we only give the auxiliary functions involved in the above inequalities. For the rest of the proof, we proceed as in Theorem 1. All the computations are done on a MacBookPro with the languages Pari and Pascal.

\section{The total distance of totally positive algebraic integers}

\subsection{The explicit auxiliary function}

Let $\alpha$ be a totally positive algebraic integer, $\alpha=\alpha_{1}, \ldots, \alpha_{d}$ be its conjugates and $P$ be its minimal polynomial. The auxiliary function involved in Theorem 1 has the following type:

$$
\text { for } x>0, f(x)=|x-1|-\sum_{0 \leq j \leq J} c_{j} \log \left|Q_{j}(x)\right|
$$

where the $c_{j}$ are positive real numbers and the polynomials $Q_{j}$ are nonzero polynomials in $\mathbb{Z}[x]$.

Then we have

$$
\sum_{i=1}^{d} f\left(\alpha_{i}\right) \geq m d
$$


where $m$ denotes the minimum of the function $f$. Thus,

$$
\operatorname{td}(\alpha) \geq m d+\sum_{1 \leq j \leq J} c_{j} \log \left|\prod_{i=1}^{d} Q_{j}\left(\alpha_{i}\right)\right|
$$

We assume that $P$ does not divide any $Q_{j}$, then $\prod_{i=1}^{d} Q_{j}\left(\alpha_{i}\right)$ is a nonzero integer because it is the resultant of $P$ and $Q_{j}$. Therefore, if $\alpha$ is not a root of $Q_{j}$, we have

$$
\operatorname{Td}(\alpha) \geq m
$$

The main problem is to find a good list of polynomials $Q_{j}$ which gives a value of $m$ as large as possible. To do this, we link the auxiliary function with a generalization of the integer transfinite diameter in order to find the polynomials with our recursive algorithm.

\subsection{Auxiliary functions and integer transfinite diameter}

In this section, we need the following definition: let $K$ be a compact subset of $\mathbb{C}$. If $\varphi$ is a positive function defined on $K$, the $\varphi$-integer transfinite diameter of $K$ is defined as

$$
\begin{aligned}
& t_{\mathbb{Z}, \varphi}(K)=\liminf \quad \inf \quad \sup |P(x)|^{\frac{1}{n}} \varphi(x) . \\
& n \geq 1 \quad P \in \mathbb{Z}[X] \quad x \in K \\
& n \rightarrow \infty \quad \operatorname{deg}(P)=n
\end{aligned}
$$

This weighted version of the integer transfinite diameter was introduced by F. Amoroso [A] and is an important tool in the study of rational approximations of logarithms of rational numbers. Inside the auxiliary function (1), we replace the numbers $c_{j}$ by rational numbers $a_{j} / q$ where $q$ is a common denominator of the $c_{j}$ for $1 \leq j \leq J$. Then we may write:

$$
\text { for } x>0, f(x)=|x-1|-\frac{t}{r} \log |Q(x)|
$$

where $Q=\prod_{j=1}^{J} Q_{j}^{a_{j}} \in \mathbb{Z}[X]$ is of degree $r=\sum_{j=1}^{J} a_{j} \operatorname{deg} Q_{j}$ and $t=\sum_{j=1}^{J} c_{j} \operatorname{deg} Q_{j}$. We search for a polynomial $Q \in \mathbb{Z}[X]$ such that

$$
\sup _{x>0}|Q(x)|^{t / r} \exp (-|x-1|) \leq e^{-m}
$$

where $m$ is as large as possible. If we suppose that $t$ is fixed, it is clear that we need an effective upper bound for the quantity

$$
\begin{aligned}
& t_{\mathbb{Z}, \varphi}((0, \infty))=\liminf _{r \geq 1} \quad \inf _{P \in \mathbb{Z}[X]} \sup _{x>0}|P(x)|^{\frac{t}{r}} \varphi(x) \\
& r \rightarrow+\infty \quad \operatorname{deg}(P)=r
\end{aligned}
$$

where we use the weight $\varphi(x)=\exp (-|x-1|)$.

Even if we replace the compact subset $K$ by the half line $(0, \infty)$, the weight $\varphi$ ensures that the quantity $t_{\mathbb{Z}, \varphi}((0, \infty))$ is finite. 


\subsection{Construction of the auxiliary function}

The polynomials involved in the auxiliary function are found by our recursive algorithm developed in [F1] from Wu's algorithm [Wu]. It replaces the heuristic search by a systematic search by induction for suitable polynomials. Suppose that we have $Q_{1}, Q_{2}, \ldots, Q_{J}$. Then we use semiinfinite linear programming (introduced into number theory by C. Smyth [Sm]) to optimize $f$ for this set of polynomials (i.e., to get the greatest possible $m$ ). We obtain the positive real numbers $c_{1}, c_{2}, \ldots, c_{J}$ and then $f$ in the form (2) as above.

For several values of $k$, we search for a polynomial $R(x)=\sum_{l=0}^{k} a_{l} x^{l} \in \mathbb{Z}[x]$ such that

$$
\sup _{x>0}|Q(x) R(x)|^{\frac{t}{r+k}} \exp (-|x-1|) \leq e^{-m}
$$

i.e., such that

$$
\sup _{x>0}|Q(x) R(x)| \exp (-|x-1|(r+k) / t)
$$

is as small as possible.

Next, we apply LLL to the linear forms

$$
Q\left(x_{i}\right) R\left(x_{i}\right) \exp \left(-\left|x_{i}-1\right|(r+k) / t\right)
$$

where the $x_{i}$ are control points in the half line $(0, \infty)$. After several trials, we find that a set of 70 points uniformly distributed in the interval $(0,70)$ is convenient for the first step of LLL. After semi-infinite linear programming, this set will be augmented by the points where $f$ has its least local minima. We get a polynomial $R$ whose factors $R_{j}$ are good candidates to enlarge the set of polynomials $\left(Q_{1}, Q_{2}, \ldots, Q_{J}\right)$. We only keep the polynomials $Q_{j}$ which have a nonzero coefficient in the newly optimized auxiliary function $f$. After optimization, some previous polynomials $Q_{j}$ may have a zero coefficient and so are removed.

In order to get the constant of Theorem 1, we take $k$ from 4 to 20 successively.

\section{Comparison of measures}

The method of explicit auxiliary functions has already been used to establish inequalities involving the Mahler measure, the length and the trace of totally positive polynomials, see [F3].

The auxiliary function used for the lower bound (resp. for the upper bound) of Theorem 2 is of the following type:

$$
\text { for } x>0, f(x)= \pm|x-1| \mp c_{0} \log \max (1, x)-\sum_{1 \leq j \leq J} c_{j} \log \left|Q_{j}(x)\right|,
$$

where the $c_{j}$ are positive real numbers and the polynomials $Q_{j}$ are nonzero polynomials in $\mathbb{Z}[x]$. The auxiliary function used for the lower bound (resp. for the upper bound) of Theorem 3 is of the following type:

$$
\text { for } x>0, f(x)= \pm \log (x+1) \mp c_{0}|x-1|-\sum_{1 \leq j \leq J} c_{j} \log \left|Q_{j}(x)\right|,
$$

where the $c_{j}$ are positive real numbers and the polynomials $Q_{j}$ are nonzero polynomials in $\mathbb{Z}[x]$. The auxiliary function used for the lower bound (resp. for the upper bound) of Theorem 4 is of the following type:

$$
\text { for } x>0, f(x)= \pm x \mp c_{0}|x-1|-\sum_{1 \leq j \leq J} c_{j} \log \left|Q_{j}(x)\right|,
$$

where the $c_{j}$ are positive real numbers and the polynomials $Q_{j}$ are nonzero polynomials in $\mathbb{Z}[x]$. 
In our recursive algorithm, we take $k$ from 4 to 10 only in order to have a small number of exceptions to the validity of the inequalities.

\section{Acknowledgments}

The author thanks the referee for his useful recommendations. 
Table 1: Polynomials $Q_{j}$ involved in Theorem 1 with their coefficients $c_{j}$

\begin{tabular}{|c|c|c|}
\hline & $c_{j}$ & Polynomial $Q_{j}$ \\
\hline 1 & 0.10655700 & $x$ \\
\hline 2 & 0.71262251 & $x-1$ \\
\hline 3 & 0.20232450 & $x-2$ \\
\hline 4 & 0.11567245 & $x^{2}-3 x+1$ \\
\hline 5 & 0.00866176 & $x^{2}-4 x+2$ \\
\hline 6 & 0.00500940 & $x^{2}-5 x+5$ \\
\hline 7 & 0.00253941 & $x^{3}-6 x^{2}+9 x-1$ \\
\hline 8 & 0.07317471 & $x^{3}-5 x^{2}+6 x-1$ \\
\hline 9 & 0.00434234 & $x^{3}-6 x^{2}+9 x-3$ \\
\hline 10 & 0.00406945 & $x^{4}-7 x^{3}+14 x^{2}-8 x+1$ \\
\hline 11 & 0.00732231 & $x^{5}-9 x^{4}+27 x^{3}-32 x^{2}+13 x-1$ \\
\hline 12 & 0.01494744 & $x^{5}-9 x^{4}+28 x^{3}-35 x^{2}+15 x-1$ \\
\hline 13 & 0.01489508 & $x^{5}-9 x^{4}+27 x^{3}-31 x^{2}+12 x-1$ \\
\hline 14 & 0.00052501 & $x^{5}-9 x^{4}+26 x^{3}-29 x^{2}+11 x-1$ \\
\hline 15 & 0.00035586 & $x^{6}-11 x^{5}+46 x^{4}-90 x^{3}+81 x^{2}-27 x+1$ \\
\hline 16 & 0.00183029 & $x^{6}-11 x^{5}+44 x^{4}-79 x^{3}+63 x^{2}-18 x+1$ \\
\hline 17 & 0.00722208 & $x^{6}-11 x^{5}+45 x^{4}-84 x^{3}+70 x^{2}-21 x+1$ \\
\hline 18 & 0.00230977 & $x^{8}-14 x^{7}+78 x^{6}-221 x^{5}+337 x^{4}-267 x^{3}+94 x^{2}-8 x-1$ \\
\hline 19 & 0.00070739 & $x^{8}-15 x^{7}+89 x^{6}-268 x^{5}+438 x^{4}-384 x^{3}+166 x^{2}-29 x+1$ \\
\hline 20 & 0.00370284 & $x^{8}-14 x^{7}+78 x^{6}-222 x^{5}+345 x^{4}-289 x^{3}+120 x^{2}-21 x+1$ \\
\hline 21 & 0.00155673 & $2 x^{8}-26 x^{7}+136 x^{6}-367 x^{5}+544 x^{4}-435 x^{3}+171 x^{2}-27 x+1$ \\
\hline 22 & 0.00217993 & $x^{9}-17 x^{8}+119 x^{7}-444 x^{6}+954 x^{5}-1188 x^{4}+817 x^{3}-276 x^{2}+37 x-1$ \\
\hline 23 & 0.00160414 & $x^{9}-17 x^{8}+119 x^{7}-444 x^{6}+954 x^{5}-1187 x^{4}+812 x^{3}-268 x^{2}+33 x-1$ \\
\hline 24 & 0.00067700 & $x^{9}-17 x^{8}+119 x^{7}-444 x^{6}+953 x^{5}-1181 x^{4}+800 x^{3}-259 x^{2}+31 x-1$ \\
\hline 25 & 0.00337174 & $x^{9}-17 x^{8}+120 x^{7}-456 x^{6}+1011 x^{5}-1324 x^{4}+986 x^{3}-376 x^{2}+57 x-1$ \\
\hline 26 & 0.00031596 & $x^{10}-18 x^{9}+135 x^{8}-550 x^{7}+1330 x^{6}-1959 x^{5}+1737 x^{4}-886 x^{3}+238 x^{2}-28 x+1$ \\
\hline 27 & 0.00753897 & $x^{10}-18 x^{9}+136 x^{8}-562 x^{7}+1388 x^{6}-2104 x^{5}+1937 x^{4}-1036 x^{3}+294 x^{2}-36 x+1$ \\
\hline 28 & 0.00415571 & $x^{10}-19 x^{9}+152 x^{8}-668 x^{7}+1764 x^{6}-2873 x^{5}+2841 x^{4}-1602 x^{3}+446 x^{2}-44 x+1$ \\
\hline 29 & 0.00152277 & $x^{10}-18 x^{9}+136 x^{8}-562 x^{7}+1387 x^{6}-2096 x^{5}+1913 x^{4}-1002 x^{3}+271 x^{2}-30 x+1$ \\
\hline 30 & 0.00038875 & $x^{10}-18 x^{9}+135 x^{8}-549 x^{7}+1320 x^{6}-1921 x^{5}+1667 x^{4}-820 x^{3}+208 x^{2}-23 x+1$ \\
\hline 31 & 0.00021183 & $x^{10}-17 x^{9}+121 x^{8}-470 x^{7}+1090 x^{6}-1556 x^{5}+1369 x^{4}-734 x^{3}+237 x^{2}-43 x+3$ \\
\hline 32 & 0.00009780 & $\begin{array}{l}x^{11}-20 x^{10}+171 x^{9}-819 x^{8}+2418 x^{7}-4560 x^{6}+5502 x^{5}-4135 x^{4}+1822 x^{3}-420 x^{2} \\
+40 x-1\end{array}$ \\
\hline 33 & 0.00012607 & $\begin{array}{l}x^{11}-21 x^{10}+189 x^{9}-954 x^{8}+2967 x^{7}-5879 x^{6}+7412 x^{5}-5760 x^{4}+2571 x^{3}-573 x^{2} \\
+46 x-1\end{array}$ \\
\hline 34 & 0.00072532 & $\begin{array}{l}x^{12}-23 x^{11}+231 x^{10}-1332 x^{9}+4878 x^{8}-11854 x^{7}+19398 x^{6}-21250 x^{5}+15190 x^{4} \\
-6738 x^{3}+1695 x^{2}-202 x+7\end{array}$ \\
\hline 35 & 0.00030507 & $\begin{array}{l}x^{12}-22 x^{11}+210 x^{10}-1142 x^{9}+3906 x^{8}-8753 x^{7}+12982 x^{6}-12579 x^{5}+7676 x^{4} \\
-2759 x^{3}+523 x^{2}-43 x+1\end{array}$ \\
\hline 36 & 0.00016745 & $\begin{array}{l}x^{12}-23 x^{11}+230 x^{10}-1314 x^{9}+4741 x^{8}-11278 x^{7}+17930 x^{6}-18906 x^{5}+12849 x^{4} \\
-5324 x^{3}+1218 x^{2}-127 x+4\end{array}$ \\
\hline 37 & 0.00145980 & $\begin{array}{l}x^{13}-24 x^{12}+252 x^{11}-1523 x^{10}+5865 x^{9}-15051 x^{8}+26160 x^{7}-30702 x^{6}+23858 x^{5} \\
-11830 x^{4}+3529 x^{3}-575 x^{2}+43 x-1\end{array}$ \\
\hline 38 & 0.00083711 & $\begin{array}{l}x^{14}-26 x^{13}+300 x^{12}-2027 x^{11}+8911 x^{10}-26781 x^{9}+56259 x^{8}-82985 x^{7}+85077 x^{6} \\
-59065 x^{5}+26496 x^{4}-7089 x^{3}+981 x^{2}-54 x+1\end{array}$ \\
\hline 39 & 0.00040496 & $\begin{array}{l}x^{14}-26 x^{13}+299 x^{12}-2006 x^{11}+8720 x^{10}-25793 x^{9}+53052 x^{8}-76192 x^{7}+75625 x^{6} \\
-50595 x^{5}+21850 x^{4}-5679 x^{3}+790 x^{2}-48 x+1\end{array}$ \\
\hline 40 & 0.00031272 & $\begin{array}{l}x^{18}-35 x^{17}+560 x^{16}-5430 x^{15}+35664 x^{14}-167990 x^{13}+586104 x^{12}-1542459 x^{11} \\
+3089299 x^{10}-4717066 x^{9}+5466151 x^{8}-4755434 x^{7}+3050835 x^{6}-1405114 x^{5}+446641 x^{4} \\
-92341 x^{3}+11292 x^{2}-691 x+15\end{array}$ \\
\hline 41 & 0.00011511 & $\begin{array}{l}x^{19}-35 x^{18}+560 x^{17}-5431 x^{16}+35692 x^{15}-168343 x^{14}+588756 x^{13}-1555701 x^{12} \\
+3135698 x^{11}-4834464 x^{10}+5683528 x^{9}-5050713 x^{8}+3343089 x^{7}-1612543 x^{6}+549476 x^{5} \\
-126561 x^{4}+18484 x^{3}-1556 x^{2}+65 x-1\end{array}$ \\
\hline 42 & 0.00042098 & $\begin{array}{l}x^{19}-35 x^{18}+560 x^{17}-5431 x^{16}+35692 x^{15}-168343 x^{14}+588756 x^{13}-1555701 x^{12} \\
+3135698 x^{11}-4834465 x^{10}+5683542 x^{9}-5050795 x^{8}+3343351 x^{7}-1613042 x^{6}+550057 x^{5} \\
-126968 x^{4}+18645 x^{3}-1587 x^{2}+67 x-1\end{array}$ \\
\hline 43 & 0.00034312 & $\begin{array}{l}x^{19}-35 x^{18}+561 x^{17}-5461 x^{16}+36098 x^{15}-171626 x^{14}+606463 x^{13}-1623033 x^{12} \\
+3321706 x^{11}-5213487 x^{10}+6255933 x^{9}-5688941 x^{8}+3862060 x^{7}-1913635 x^{6}+669910 x^{5} \\
-158075 x^{4}+23448 x^{3}-1962 x^{2}+77 x-1\end{array}$ \\
\hline 44 & 0.00085592 & $\begin{array}{l}x^{19}-35 x^{18}+560 x^{17}-5431 x^{16}+35692 x^{15}-168344 x^{14}+588778 x^{13}-1555913 x^{12} \\
+3136877 x^{11}-4838661 x^{10}+5693555 x^{9}-5067109 x^{8}+3361466 x^{7}-1626464 x^{6}+556386 x^{5} \\
-128692 x^{4}+18858 x^{3}-1588 x^{2}+66 x-1\end{array}$ \\
\hline 45 & 0.00124561 & $\begin{array}{l}x^{19}-35 x^{18}+561 x^{17}-5461 x^{16}+36099 x^{15}-171649 x^{14}+606698 x^{13}-1624441 x^{12} \\
+3327206 x^{11}-5228221 x^{10}+6283668 x^{9}-5725945 x^{8}+3896983 x^{7}-1936732 x^{6}+680478 x^{5} \\
-161381 x^{4}+24145 x^{3}-2055 x^{2}+83 x-1\end{array}$ \\
\hline 46 & 0.00031896 & $\begin{array}{l}x^{19}-35 x^{18}+561 x^{17}-5460 x^{16}+36071 x^{15}-171298 x^{14}+604092 x^{13}-1611668 x^{12} \\
+3283605 x^{11}-5121678 x^{10}+6095068 x^{9}-5483972 x^{8}+3674067 x^{7}-1792070 x^{6}+616291 x^{5} \\
-142721 x^{4}+20802 x^{3}-1722 x^{2}+69 x-1\end{array}$ \\
\hline
\end{tabular}


Table 2: Comparison of the bounds of Stulov and Yang and the author's bounds. Here $P$ denotes the minimal polynomial of a totally positive algebraic integer of degree $d$.

The lower bound of Stulov and Yang
The author's lower bound
The author's upper bound
The upper bound of Stulov and Yang

\begin{tabular}{|c|c|c|c|c|c|c|}
\hline$P$ & $\mathrm{~m}(P)$ & 0.227804 & $\mathrm{~m}(P)+1.077638 d$ & $\operatorname{td}(P)$ & $86.546972 \mathrm{~m}(P)-43.686533 d$ & $2 \mathrm{M}(P)-1$ \\
\hline$P_{1}$ & 2.1678737 & & 4.8044023 & 5.3307043 & 12.876775 & 15.479363 \\
\hline$P_{2}$ & 2.0879857 & & 4.7862035 & 5.4562194 & 5.9627053 & 14.137292 \\
\hline$P_{3}$ & 2.8102287 & & 6.0283713 & 6.5877984 & 24.784124 & 31.227436 \\
\hline$P_{4}$ & 2.8837927 & & 6.0451295 & 6.4574148 & 31.150865 & 33.763933 \\
\hline$P_{5}$ & 2.8096787 & & 6.0282461 & 6.7109713 & 24.736521 & 31.209166 \\
\hline$P_{6}$ & 2.7481251 & & 6.0142239 & 6.7611431 & 19.409241 & 29.226662 \\
\hline$P_{7}$ & 3.4600638 & & 7.2540444 & 7.9852747 & 37.338850 & 61.638016 \\
\hline$P_{8}$ & 3.5325158 & & 7.2705492 & 7.8778103 & 43.609351 & 66.419852 \\
\hline$P_{9}$ & 3.3692819 & & 7.2333639 & 8.4370661 & 29.481952 & 56.115309 \\
\hline$P_{10}$ & 4.0647148 & & 8.4694243 & 10.133815 & 45.983024 & 114.49658 \\
\hline$P_{11}$ & 4.1344755 & & 8.4853161 & 9.9760434 & 52.020603 & 122.91365 \\
\hline$P_{12}$ & 4.6480352 & & 9.6799450 & 10.973399 & 52.781105 & 206.75939 \\
\hline$P_{13}$ & 4.4586497 & & 9.6368022 & 10.172583 & 36.390369 & 170.74161 \\
\hline$P_{14}$ & 5.4858197 & & 10.948434 & 12.480484 & 81.602289 & 480.49324 \\
\hline$P_{15}$ & 5.4175259 & & 10.932876 & 11.962019 & 75.691664 & 448.64192 \\
\hline$P_{16}$ & 5.4904408 & & 10.949486 & 12.464721 & 82.002232 & 482.72805 \\
\hline$P_{17}$ & 5.5064050 & & 10.953123 & 12.519260 & 83.383879 & 490.52841 \\
\hline$P_{18}$ & 6.0204376 & & 12.147860 & 13.500532 & 84.185315 & 821.51749 \\
\hline$P_{19}$ & 5.6605471 & & 12.065875 & 13.366620 & 53.037882 & 572.61157 \\
\hline$P_{20}$ & 5.5713426 & & 12.045554 & 13.433823 & 45.317500 & 523.57335 \\
\hline$P_{21}$ & 5.5661460 & & 12.044370 & 13.488010 & 44.867748 & 520.84922 \\
\hline$P_{22}$ & 6.8257165 & & 14.486582 & 16.412179 & 66.506699 & 1840.4725 \\
\hline$P_{23}$ & 6.8465121 & & 14.491319 & 16.464912 & 68.306499 & 1879.1890 \\
\hline$P_{24}$ & 7.6494198 & & 15.751862 & 17.801539 & 94.109190 & 4196.8542 \\
\hline$P_{25}$ & 8.2171482 & & 16.958831 & 18.935244 & 99.557834 & 7405.8489 \\
\hline$P_{26}$ & 8.9419509 & & 19.279220 & 21.778250 & 74.914243 & 15290.198 \\
\hline$P_{27}$ & 11.016533 & & 22.984732 & 25.502603 & 123.40342 & 121742.50 \\
\hline
\end{tabular}

where

$P_{1}=x^{4}-7 x^{3}+14 x^{2}-8 x+1$

$P_{2}=x^{4}-7 x^{3}+13 x^{2}-7 x+1$

$P_{3}=x^{5}-9 x^{4}+27 x^{3}-32 x^{2}+13 x-1$

$P_{4}=x^{5}-9 x^{4}+28 x^{3}-35 x^{2}+15 x-1$

$P_{5}=x^{5}-9 x^{4}+27 x^{3}-31 x^{2}+12 x-1$

$P_{6}=x^{5}-9 x^{4}+26 x^{3}-29 x^{2}+11 x-1$

$P_{7}=x^{6}-11 x^{5}+44 x^{4}-79 x^{3}+63 x^{2}-18 x+1$

$P_{8}=x^{6}-11 x^{5}+45 x^{4}-84 x^{3}+70 x^{2}-21 x+1$

$P_{9}=x^{6}-11 x^{5}+43 x^{4}-72 x^{3}+51 x^{2}-14 x+1$

$P_{10}=x^{7}-13 x^{6}+63 x^{5}-143 x^{4}+157 x^{3}-78 x^{2}+16 x-1$

$P_{11}=x^{7}-13 x^{6}+64 x^{5}-150 x^{4}+172 x^{3}-89 x^{2}+18 x-1$

$P_{12}=x^{8}-15 x^{7}+89 x^{6}-268 x^{5}+438 x^{4}-384 x^{3}+166 x^{2}-29 x+1$

$P_{13}=x^{8}-14 x^{7}+78 x^{6}-221 x^{5}+337 x^{4}-267 x^{3}+94 x^{2}-8 x-1$

$P_{14}=x^{9}-17 x^{8}+119 x^{7}-444 x^{6}+954 x^{5}-1188 x^{4}+817 x^{3}-276 x^{2}+37 x-1$

$P_{15}=x^{9}-17 x^{8}+120 x^{7}-456 x^{6}+1011 x^{5}-1324 x^{4}+986 x^{3}-376 x^{2}+57 x-1$

$P_{16}=x^{9}-17 x^{8}+119 x^{7}-444 x^{6}+954 x^{5}-1187 x^{4}+812 x^{3}-268 x^{2}+33 x-1$

$P_{17}=x^{9}-17 x^{8}+119 x^{7}-444 x^{6}+953 x^{5}-1181 x^{4}+800 x^{3}-259 x^{2}+31 x-1$

$P_{18}=x^{10}-19 x^{9}+152 x^{8}-668 x^{7}+1764 x^{6}-2873 x^{5}+2841 x^{4}-1602 x^{3}+446 x^{2}-44 x+1$

$P_{19}=x^{10}-18 x^{9}+135 x^{8}-549 x^{7}+1320 x^{6}-1920 x^{5}+1662 x^{4}-813 x^{3}+206 x^{2}-24 x+1$

$P_{20}=x^{10}-18 x^{9}+134 x^{8}-538 x^{7}+1273 x^{6}-1822 x^{5}+1560 x^{4}-766 x^{3}+200 x^{2}-24 x+1$

$P_{21}=x^{10}-18 x^{9}+134 x^{8}-537 x^{7}+1265 x^{6}-1798 x^{5}+1526 x^{4}-743 x^{3}+194 x^{2}-24 x+1$

$P_{22}=x^{12}-22 x^{11}+208 x^{10}-1108 x^{9}+3667 x^{8}-7851 x^{7}+10995 x^{6}-9977 x^{5}+5702 x^{4}$ $-1952 x^{3}+368 x^{2}-33 x+1$

$P_{23}=x^{12}-22 x^{11}+208 x^{10}-1108 x^{9}+3666 x^{8}-7840 x^{7}+10948 x^{6}-9877 x^{5}+5589 x^{4}$ $-1885 x^{3}+349 x^{2}-31 x+1$

$P_{24}=x^{13}-24 x^{12}+252 x^{11}-1523 x^{10}+5865 x^{9}-15051 x^{8}+26160 x^{7}-30702 x^{6}+23858 x^{5}$ $-11830 x^{4}+3529 x^{3}-575 x^{2}+43 x-1$

$P_{25}=x^{14}-26 x^{13}+299 x^{12}-2006 x^{11}+8720 x^{10}-25793 x^{9}+53052 x^{8}-76192 x^{7}+75625 x^{6}$ $-50595 x^{5}+21850 x^{4}-5679 x^{3}+790 x^{2}-48 x+1$

$P_{26}=x^{16}-29 x^{15}+375 x^{14}-2859 x^{13}+14320 x^{12}-49711 x^{11}+123001 x^{10}-219813 x^{9}$

$+284591 x^{8}-265614 x^{7}+176517 x^{6}-81849 x^{5}+25677 x^{4}-5199 x^{3}+630 x^{2}-40 x+1$
$P_{27}=x^{19}-35 x^{18}+560 x^{17}-5431 x^{16}+35692 x^{15}-168343 x^{14}+588756 x^{13}-1555701 x^{12}$ $+3135698 x^{11}-4834465 x^{10}+5683542 x^{9}-5050795 x^{8}+3343351 x^{7}-1613042 x^{6}$ $+550057 x^{5}-126968 x^{4}+18645 x^{3}-1587 x^{2}+67 x-1$ 
Table 3: Comparison of the trace and the total distance of some polynomials $P$ which are minimal polynomials of totally positive algebraic integers of degree $d$.

\begin{tabular}{|c|c|c|c|c|}
\hline$P$ & 0.236643 & $\operatorname{td}(P)+1.077638 d$ & $\operatorname{trace}(P)$ & $2.211970 \operatorname{td}(P)-0.635402 d$ \\
\hline$P_{1}$ & & 6.4927498 & 7 & 9.7538396 \\
\hline$P_{2}$ & & 6.5224521 & 7 & 10.113062 \\
\hline$P_{3}$ & & 8.0980514 & 9 & 11.975985 \\
\hline$P_{4}$ & & 8.0671970 & 9 & 11.602829 \\
\hline$P_{5}$ & & 8.1271994 & 9 & 12.328504 \\
\hline$P_{6}$ & & 8.1390722 & 9 & 12.472095 \\
\hline$P_{7}$ & & 9.7365734 & 11 & 14.599902 \\
\hline$P_{8}$ & & 9.7111427 & 11 & 14.292340 \\
\hline$P_{9}$ & & 9.8434866 & 11 & 15.892924 \\
\hline$P_{10}$ & & 11.552829 & 13 & 19.373356 \\
\hline$P_{11}$ & & 11.515494 & 13 & 18.921816 \\
\hline$P_{12}$ & & 13.059330 & 15 & 20.400591 \\
\hline$P_{13}$ & & 12.869823 & 14 & 18.108666 \\
\hline$P_{14}$ & & 14.723790 & 17 & 23.338209 \\
\hline$P_{15}$ & & 14.601099 & 17 & 21.854367 \\
\hline$P_{16}$ & & 14.720060 & 17 & 23.293094 \\
\hline$P_{17}$ & & 14.732966 & 17 & 23.449186 \\
\hline$P_{18}$ & & 16.272996 & 19 & 24.881930 \\
\hline$P_{19}$ & & 16.241307 & 18 & 24.498675 \\
\hline$P_{20}$ & & 16.257210 & 18 & 24.691011 \\
\hline$P_{21}$ & & 16.270033 & 18 & 24.846094 \\
\hline$P_{22}$ & & 19.577655 & 22 & 30.463744 \\
\hline$P_{23}$ & & 19.590134 & 22 & 30.614665 \\
\hline$P_{24}$ & & 21.214257 & 24 & 33.064432 \\
\hline$P_{25}$ & & 22.790359 & 26 & 34.933438 \\
\hline$P_{26}$ & & 26.078774 & 29 & 40.318803 \\
\hline$P_{27}$ & & 30.883573 & 35 & 46.850926 \\
\hline
\end{tabular}

Table 4: Comparison of the length and the total distance of some polynomials $P$ which are minimal polynomials of totally positive algebraic integers of degree $d$.

\begin{tabular}{|c|c|c|c|c|}
\hline$P$ & 0.236643 & $\operatorname{td}(P)+1.077638 \mathrm{~d}$ & $\operatorname{trace}(P)$ & $2.211970 \operatorname{td}(P)-0.635402 d$ \\
\hline$P_{1}$ & & 2.8962059 & 3.4339872 & 5.1854808 \\
\hline$P_{2}$ & & 2.8994896 & 3.3672958 & 5.3216642 \\
\hline$P_{3}$ & & 3.6182800 & 4.4188406 & 6.3998449 \\
\hline$P_{4}$ & & 3.6148689 & 4.4886364 & 6.2583793 \\
\hline$P_{5}$ & & 3.6215024 & 4.3944492 & 6.5334870 \\
\hline$P_{6}$ & & 3.6228150 & 4.3438054 & 6.5879232 \\
\hline$P_{7}$ & & 4.3440268 & 5.3798974 & 7.7665231 \\
\hline$P_{8}$ & & 4.3412153 & 5.4510385 & 7.6499246 \\
\hline$P_{9}$ & & 4.3558465 & 5.2626902 & 8.2567150 \\
\hline$P_{10}$ & & 5.0894229 & 6.1569790 & 9.9481027 \\
\hline$P_{11}$ & & 5.0852952 & 6.2304814 & 9.7769212 \\
\hline$P_{12}$ & & 5.8005741 & 7.2377782 & 10.709470 \\
\hline$P_{13}$ & & 5.7796231 & 6.9265770 & 9.8405877 \\
\hline$P_{14}$ & & 6.5291884 & 8.2568668 & 12.195073 \\
\hline$P_{15}$ & & 6.5156243 & 8.3777012 & 11.632541 \\
\hline$P_{16}$ & & 6.5287760 & 8.2521854 & 12.177970 \\
\hline$P_{17}$ & & 6.5302029 & 8.2443340 & 12.237145 \\
\hline$P_{18}$ & & 7.2450609 & 9.2506182 & 13.152243 \\
\hline$P_{19}$ & & 7.2415575 & 8.8022217 & 13.006949 \\
\hline$P_{20}$ & & 7.2433157 & 8.7541607 & 13.079865 \\
\hline$P_{21}$ & & 7.2447333 & 8.7388957 & 13.138657 \\
\hline$P_{22}$ & & 8.6996074 & 10.642683 & 16.012213 \\
\hline$P_{23}$ & & 8.7009870 & 10.634051 & 16.069428 \\
\hline$P_{24}$ & & 9.4251419 & 11.690352 & 17.370085 \\
\hline$P_{25}$ & & 10.143988 & 12.678190 & 18.450572 \\
\hline$P_{26}$ & & 11.596739 & 14.038836 & 21.236066 \\
\hline$P_{27}$ & & 13.761733 & 17.100651 & 24.828240 \\
\hline
\end{tabular}


Table 5: Comparison of the bounds of Stulov and Yang and the author's for some reciprocal polynomials $P$ which are minimal polynomials of totally positive reciprocal algebraic integers of degree $d$.

The improved lower bound of Stulov and Yang
The author's lower bound
The author's upper bound
The upper bound of Stulov and Yang

\begin{tabular}{|c|c|c|c|c|c|}
\hline$P$ & $2 \mathrm{~m}(P)$ & $0.227804 \mathrm{~m}(P)+1.077638 \mathrm{~d}$ & $\operatorname{td}(P)$ & $86.546972 \mathrm{~m}(P)-43.686533 \mathrm{~d}$ & $2 \mathrm{M}(P)-1$ \\
\hline$P_{1}$ & 4.1759713 & 5.2134895 & 5.4562194 & 5.9627053 & 14.137292 \\
\hline$P_{2}$ & 4.4523454 & 5.4285522 & 6.4412053 & 17.922373 & 16.528681 \\
\hline$P_{3}$ & 6.4766953 & 7.9857783 & 8.7017102 & 18.149987 & 48.983133 \\
\hline$P_{4}$ & 6.5241080 & 8.0226728 & 9.6390906 & 20.201699 & 50.206196 \\
\hline$P_{5}$ & 8.5857452 & 10.608914 & 11.898492 & 22.042862 & 144.35275 \\
\hline$P_{6}$ & 8.7932213 & 10.770363 & 12.885760 & 31.021073 & 160.35054 \\
\hline$P_{7}$ & 8.6714949 & 10.675641 & 11.919817 & 25.753549 & 150.76406 \\
\hline$P_{8}$ & 8.7121732 & 10.707295 & 12.864449 & 27.513840 & 153.90296 \\
\hline$P_{9}$ & 13.076541 & 16.067387 & 18.359886 & 41.629114 & 1380.1805 \\
\hline$P_{10}$ & 15.285923 & 18.768598 & 21.581474 & 49.863733 & 4169.8252 \\
\hline$P_{11}$ & 15.332635 & 18.804946 & 21.602688 & 51.885084 & 4268.4072 \\
\hline$P_{12}$ & 15.254839 & 18.744409 & 21.582923 & 48.518583 & 4105.4862 \\
\hline$P_{13}$ & 15.265027 & 18.752337 & 21.585202 & 48.959471 & 4126.4639 \\
\hline$P_{14}$ & 17.355512 & 21.361027 & 24.785407 & 52.048999 & 11739.718 \\
\hline$P_{15}$ & 17.451738 & 21.435906 & 24.801640 & 56.213016 & 12318.456 \\
\hline$P_{16}$ & 24.134495 & 29.582033 & 34.490281 & 83.280024 & 348150.16 \\
\hline$P_{17}$ & 28.588126 & 35.011585 & 40.931773 & 101.25801 & 3227471.5 \\
\hline
\end{tabular}

where

$P_{1}=x^{4}-7 x^{3}+13 x^{2}-7 x+1$

$P_{2}=x^{4}-8 x^{3}+15 x^{2}-8 x+1$

$P_{3}=x^{6}-11 x^{5}+41 x^{4}-63 x^{3}+41 x^{2}-11 x+1$

$P_{4}=x^{6}-12 x^{5}+44 x^{4}-67 x^{3}+44 x^{2}-12 x+1$

$P_{5}=x^{8}-15 x^{7}+83 x^{6}-220 x^{5}+303 x^{4}-220 x^{3}+83 x^{2}-15 x+1$

$P_{6}=x^{8}-16 x^{7}+91 x^{6}-244 x^{5}+337 x^{4}-244 x^{3}+91 x^{2}-16 x+1$

$P_{7}=x^{8}-15 x^{7}+84 x^{6}-225 x^{5}+311 x^{4}-225 x^{3}+84 x^{2}-15 x+1$

$P_{8}=x^{8}-16 x^{7}+90 x^{6}-239 x^{5}+329 x^{4}-239 x^{3}+90 x^{2}-16 x+1$

$P_{9}=x^{12}-23 x^{11}+218 x^{10}-1118 x^{9}+3438 x^{8}-6651 x^{7}+8271 x^{6}-6651 x^{5}+3438 x^{4}-1118 x^{3}+218 x^{2}-23 x+1$

$P_{10}=x^{14}-27 x^{13}+309 x^{12}-1977 x^{11}+7871 x^{10}-20564 x^{9}+36261 x^{8}-43749 x^{7}+36261 x^{6}-20564 x^{5}+7871 x^{4}$ $-1977 x^{3}+309 x^{2}-27 x+1$

$P_{11}=x^{14}-27 x^{13}+309 x^{12}-1979 x^{11}+7894 x^{10}-20668 x^{9}+36503 x^{8}-44067 x^{7}+36503 x^{6}-20668 x^{5}+7894 x^{4}$ $-1979 x^{3}+309 x^{2}-27 x+1$

$P_{12}=x^{14}-27 x^{13}+308 x^{12}-1963 x^{11}+7790 x^{10}-20307 x^{9}+35763 x^{8}-43131 x^{7}+35763 x^{6}-20307 x^{5}+7790 x^{4}$ $-1963 x^{3}+308 x^{2}-27 x+1$

$P_{13}=x^{14}-27 x^{13}+308 x^{12}-1964 x^{11}+7800 x^{10}-20348 x^{9}+35853 x^{8}-43247 x^{7}+35853 x^{6}-20348 x^{5}+7800 x^{4}$ $-1964 x^{3}+308 x^{2}-27 x+1$

$P_{14}=x^{16}-31 x^{15}+413 x^{14}-3141 x^{13}+15261 x^{12}-50187 x^{11}+115410 x^{10}-189036 x^{9}+222621 x^{8}-189036 x^{7}$ $+115410 x^{6}-50187 x^{5}+15261 x^{4}-3141 x^{3}+413 x^{2}-31 x+1$

$P_{15}=x^{16}-31 x^{15}+415 x^{14}-3177 x^{13}+15538 x^{12}-51389 x^{11}+118680 x^{10}-194903 x^{9}+229733 x^{8}-194903 x^{7}$ $+118680 x^{6}-51389 x^{5}+15538 x^{4}-3177 x^{3}+415 x^{2}-31 x+1$

$P_{16}=x^{22}-43 x^{21}+832 x^{20}-9625 x^{19}+74627 x^{18}-412074 x^{17}+1680988 x^{16}-5187203 x^{15}+12299616 x^{14}-22643382 x^{13}$ $+32578283 x^{12}-36764041 x^{11}+32578283 x^{10}-22643382 x^{9}+12299616 x^{8}-5187203 x^{7}+1680988 x^{6}-412074 x^{5}$ $+74627 x^{4}-9625 x^{3}+832 x^{2}-43 x+1$

$P_{17}=x^{26}-51 x^{25}+1191 x^{24}-16934 x^{23}+164453 x^{22}-1160191 x^{21}+6175143 x^{20}-25425203 x^{19}+82394564 x^{18}$ $-212746984 x^{17}+441495979 x^{16}-740812263 x^{15}+1009086208 x^{14}-1118311827 x^{13}+1009086208 x^{12}-740812263 x^{11}$ $+441495979 x^{10}-212746984 x^{9}+82394564 x^{8}-25425203 x^{7}+6175143 x^{6}-1160191 x^{5}+164453 x^{4}-16934 x^{3}$ $+1191 x^{2}-51 x+1$ 


\section{References}

[ABP] J. Aguirre, M. Bilbao, J. C. Peral. The trace of totally positive algebraic integers, Math. Comp.,75 (2006), no 253, 385-393.

[A] F. Amoroso. f-transfinite diameter and number theoretic applications, Ann. Inst. Fourier, Grenoble 43 (1993), 1179-1198.

[CS] S. Choi, C. Samuels. Two inequalities on the areal Mahler measure, Illinois J. Math. 56 (2012), no. 3, 825-834.

[F1] V. Flammang. On the absolute trace of polynomials having all zeros in a sector, Experiment. Math. 17 (2008), no. 4, 443-450.

[F2] V. Flammang. Trace of totally positive algebraic integers and integer transfinite diameter, Math. Comp. 78 (2009), no. 266, 1119-1125.

[F3] V. Flammang. Comparison of measures of totally positive polynomials, Bull. Aust. Math. Soc. 89 (2014), no. 2, 265-270.

[FR] V. Flammang, G. Rhin. On the absolute Mahler measure of polynomials having all zeros in a sector III, to appear in Math. Comp.

[F4] V. Flammang. Construction de fonctions auxiliaires. Application aux mesures de certaines familles d'entiers algébriques, in preparation.

[H] H. Huang. Mahler's measures on function spaces, Illinois J. Math. 55 (2011), no. 3, $1183-1202$.

[K] L. Kronecker. Zwei Sätze über Gleichugen mit ganzzahligen Koeffizienten, J. reine angew. Math. 53 (1857), 173-175.

[L] D. H. Lehmer. Factorization of certain cyclotomic functions, Ann. of Math. (2) 34 (1933), no. $3,461-479$.

[La] M. Langevin. Minorations de la maison et de la mesure de Mahler de certains entiers algébriques, C. R. Acad. Sci. Paris, 303 (1986), 523-526.

[RS] G. Rhin, C.J. Smyth. On the absolute Mahler measure of polynomials having all zeros in a sector, Math. Comp. 64 (209) (1995), 295-304.

[RW] G. Rhin, Q. Wu. On the absolute Mahler measure of polynomials having all zeros in a sector II, Math. Comp. 74 (249)(2004), 383-388.

[Sc] I. Schur. Uber die Verteilung der Wurzeln bei gewissen algebraischen Gleichungen mit ganzzahligen Koeffizienten, Math. Z. 1 (1918), 377-402.

[Si] C.L. Siegel. The trace of totally positive and real algebraic integers, Ann. of Maths 46 (1945), 302-312.

[Sm] C.J. Smyth. The mean value of totally real algebraic numbers, Math. Comp. 42 (1984), 663-681.

[SY] K. Stulov, R. Yang. An elementary inequality about the Mahler measure, Involve 6 (2013), no. 4, 393-397.

[Wu] Q. Wu. On the linear independence measure of logarithms of rational numbers, Math. Comp. 72 (2003), 901-911.

[WW] Q. Wu, L. Wang. On the irrationality measure of $\log$ 3, J. Number Theory 142 (2014), 264-273. 
UMR CNRS 7502. IECL, Université de Lorraine, site de Metz, Département de Mathématiques, UFR MIM, Ile du Saulcy, CS 50128. 57045 METZ cedex 01. FRANCE

E-mail address :valerie.flammang@univ-lorraine.fr 